\title{
The role of cardiac rehabilitation after Streptococcus agalactiae Infective Endocarditis with Septic Embolic Complication in an Elderly Patient: Case Report
}

\author{
OPREA Alexandru ${ }^{1,2}$, SCURTU Razvan ${ }^{1,3}$, ENCICA Svetlana ${ }^{2}$, LAPUSAN Emanuela ${ }^{4}$, \\ SERBAN Adela ${ }^{1,2}$, SACUI Diana ${ }^{2}$, BLENDEA Dan ${ }^{1,3}$
}

Editor: Constantin MUNTEANU, E-mail: office@bioclima.ro

Corresponding author: SACUI Diana, E-mail: diana sacui27@yahoo.com,

1.’Iuliu Hatieganu University of Medicine and Pharmacy" Cluj-Napoca, Romania 2."Niculae Stancioiu Emergency Heart Institute for Cardiovascular Diseases" Cluj-Napoca, Romania 3. "Emergency Clinic County Hospital", Cluj-Napoca, Romania 4."Polaris Medical Clinic", Suceagu, Romania

\section{Abstract}

Introduction. In the past, Streptococcus agalactiae was known to be associated with invasive infections in pregnant women and newborns. More recently, given a more appropriate antibiotic prophylaxis and treatment, the incidence among pregnant women and infants diminished. However, an increasing number of cases with infective endocarditis with Streptococcus agalactiae has been reported in the recent years in older patients with underlying comorbidities.

Case report. An 80-year-old female patient presented with dyspnea, weight loss, fever, fatigue, chills, dry cough and bilateral lower limb edema. The work-up revealed complicated infective endocarditis with Streptococcus agalactiae on the native aortic valve with severe aortic regurgitation as a result of the valvular destruction and a metastatic spleen abscess. Antibiotic treatment was started with resolution of the fever and chills. The patient however developed congestive heart failure due to the valvular regurgitation. A decision was made to proceed urgently with aortic valve replacement and splenectomy, which were performed at the same time, followed by a good recovery.

Results and discussion. An increasing number of cases with IE with GBS in elderly patients has been reported in the recent years, with high rate of complications and mortality. The underlying comorbidities are important risk factors for S. agalactiae IE. Considering our case, a patient in her eighties with significant abdominal surgical history and multiple medical conditions could be a typical host. Recovery after cardiac surgery is one of the most important indications of physical training. This includes patients post-coronary artery bypass grafting, after valve prosthetic replacement, after surgery for congenital diseases and after heart transplantation. Early mobilization is particularly important in avoiding immobility and cardiac deconditioning.

Conclusions. Despite the fact that mortality in Group B Streptococcus endocarditis is $40 \%$, a combined medical and surgical strategy individualized to the specific situation of each patient have led to a positive outcome in a number of cases. We present such a combined treatment approach in a case of complicated infective endocarditis with metastatic spleen abscess in an elderly patient. The aortic valve replacement and splenectomy were performed at the same time.

Keywords: endocarditis, septic emboli, valve replacement, splenectomy,

\section{INTRODUCTION}

In the past, Group B Streptococcus agalactiae (GBS) used to be associated with invasive infections in pregnant women and newborns $(\mathbf{1}, \mathbf{2})$.

Streptococcus agalactiae, a gram-positive bacteria, is often found in gastrointestinal, genital and upper respiratory tracts (2). In recent years, due to antibiotic prophylaxis and treatment, the incidence among pregnant women and infants has significantly diminished (2). This has not been the case with other infections with GBS. An increasing number of infective endocarditis (IE) with S. agalactiae has been reported in older patients which have underlying comorbidities, such as diabetes, cardiovascular disease, malignancy, advanced hepatic and renal disease $(\mathbf{2}, \mathbf{3})$. According to some studies, mortality in GBS endocarditis is $40 \%$ (4), but a combined medical and surgical strategy individualized to the specific situation of each patient have led to a positive outcome in a number of cases.

According to a study by Farley et al., almost 9\% of positive GBS blood cultures from patients with 
bacteriemia have endocarditis (5). It was observed that infective endocarditis with S. agalactiae occurs mainly on native left-sided valves, rather than right side.

\section{Case report}

We report a case of an 80-year-old female who presented with fever, chills, fatigue, dry cough, dyspnea, weight loss (20 kilograms in one year) and bilateral lower extremity edema, with the onset 3-4 months prior to the admission. Her past medical history included hypertension, Vaterian ampulloma for which cephalic duodeno-pancreatectomy was performed (2014), exocrine pancreatic insufficiency, cholecystectomy, uterine leiomyoma, moderate anemia, nodular thyroid hyperplasia, without other significant family or personal history. Her chronic treatment included: a beta-blocker, an ACE inhibitor, a thiazide-like diuretic, loop diuretic, aldosterone antagonist, HMG-CoA reductase inhibitor, pancreatic enzyme supplement. On examination the Body Mass Index (BMI) was $21 \mathrm{~kg} / \mathrm{m}^{2}$, the temperature was $38.3^{\circ} \mathrm{C}$, blood pressure $145 / 50 \mathrm{mmHg}$, heart rate 85 beats per minute, oxygen saturation $89 \%$ in ambient air and $94 \%$ with 21 of oxygen via nasal canula, the respiratory rate $24 / \mathrm{min}$, jugular venous pressure $6 \mathrm{~cm} \mathrm{H}_{2} \mathrm{O}$. To note the skin had no pathological modifications other than pallor. There were diminished breath sounds at both bases. No bruits were heard in the carotid, subclavian, or femoral arteries, and the peripheral pulses were 2+ throughout. The first and second heart sounds (s1, s2) had regular rate and rhythm, and no gallops (s3, s4) were audible. There was a grade $2 / 6$ holodiastolic decrescendo murmur at the left lower sternal border and a $2 / 6$ holosystolic murmur at the apex radiating towards the axilla. and bilateral lower limb edema. Examination was also notable for hepatosplenomegaly, with the liver and spleen palpable approximately $2 \mathrm{~cm}$ below the costal margin. The remainder of the examination was normal.

Laboratory tests (see Table 1) revealed elevated inflammatory markers, increased serum creatinine and urea with a glomerular filtration rate (GFR) of 19 $\mathrm{ml} / \mathrm{min} / 1.73 \mathrm{~m}^{2}$, moderate hypoproteinemia with hypoalbuminemia, moderate normochromic, normocytic anemia and altered coagulation parameters.

Table 1. Laboratory tests.

\begin{tabular}{|l|c|c|}
\hline Parameters & $\begin{array}{l}\text { Reference } \\
\text { range }\end{array}$ & $\begin{array}{l}\text { On } \\
\text { presentation }\end{array}$ \\
\hline Hemoglobin $(\mathrm{g} / \mathrm{dl})$ & $12-15.5$ & $\mathbf{8 . 7}$ \\
\hline Hematocrit $(\%)$ & $37-47$ & $\mathbf{2 5 . 5}$ \\
\hline $\begin{array}{l}\text { White blood cell count } \\
(/ \mathrm{mm} 3)\end{array}$ & $4-10$ & $\mathbf{1 2 . 3 1}$ \\
\hline Platelet count $(/ \mathrm{mm} 3)$ & $150-400$ & 153 \\
\hline Sodium $(\mathrm{mmol} / \mathrm{l})$ & $136-146$ & $\mathbf{1 3 0}$ \\
\hline Potassium $(\mathrm{mmol} / \mathrm{l})$ & $3.5-5.1$ & 4.34 \\
\hline Chloride $(\mathrm{mmol} / \mathrm{l})$ & $101-109$ & 103 \\
\hline Urea nitrogen $(\mathrm{mg} / \mathrm{d})$ & $17-43$ & $\mathbf{1 7 0}$ \\
\hline
\end{tabular}

\begin{tabular}{|l|c|c|}
\hline $\begin{array}{l}\text { Serum creatinine } \\
(\mathrm{mg} / \mathrm{dl})\end{array}$ & $0.51-0.95$ & $\mathbf{2 . 2 7}$ \\
\hline Glucose $(\mathrm{mg} / \mathrm{dl})$ & $74-106$ & $\mathbf{1 4 1}$ \\
\hline
\end{tabular}

Urinalysis showed microscopic proteinuria, hematuria, and leukocyturia, so a quantitative test for proteinuria was made, which revealed $726 \mathrm{mg}$ proteins per $24 \mathrm{~h}$. In addition, urine culture found Escherichia Coli and the patient got Fosfomycin in unique dose, with further negative cultures.

The electrocardiogram revealed sinus rhythm, with a ventricular rate of 90 beats per minute, QRS axis at $-30^{\circ}$, left ventricular hypertrophy, poor $\mathrm{R}$ wave progression in V1-V3.

A bedside transthoracic echocardiography (TTE) showed a vegetation on the right coronary cusp of the aortic valve and severe aortic insufficiency, and the left ventricular ejection fraction of $51 \%$.

Considering the clinical presentation, empiric antimicrobial therapy was initiated with Ampicillin, Oxacillin and Gentamicin after the blood cultures were drawn. The cultures were positive for Streptococcus agalactiae. As a result the antimicrobial treatment was continued with a combination of Ampicillin and Gentamicin.

Given the patient's symptoms, the laboratory findings and positive blood culture a transesophageal echocardiography (TEE) was performed, and revealed at the right coronary cusp of the aortic valve a $15 / 7 / 8 \mathrm{~mm}$ hyperechoic mobile mass (Figure 1). Also, severe aortic regurgitation (pressure half time (PHT) $=157 \mathrm{~ms}$, vena contracta $=6 \mathrm{~mm}$ ) with eccentric jet was noticed as a cause of the valvular destruction (Figure 2).

During hospitalization, the patient had several episodes of acute left sided heart failure with pulmonary edema in the setting of worsening aortic regurgitation and acute on chronic kidney failure (GFR decreased to $11 \mathrm{ml} / \mathrm{min} / 1.73$ $\mathrm{m}^{2}$ ). Cardiac surgery and general surgery consultations were requested and a decision was made to proceed urgently with aortic valve replacement and splenectomy.

Thirteen days after the initial presentation, the patient was transferred to our department of cardio- vascular surgery due to episodes of acute left side heart failure with pulmonary edema in the setting of worsening aortic regurgitation and acute on chronic kidney failure.

The case was discussed by the Endocarditis Heart Team of our hospital, and with a general surgeon, considering the large size of the vegetation $(>1.5 \mathrm{~cm})$, the onset of clinical signs of heart failure, acute pulmonary edema, and acute kidney failure, an indication for surgical aortic valve replacement and splenectomy was made. The patient's frailness, the multiple comorbidities and the high operative risk entailed the performance of the two proposed urgent surgical procedures at the same operative time. 


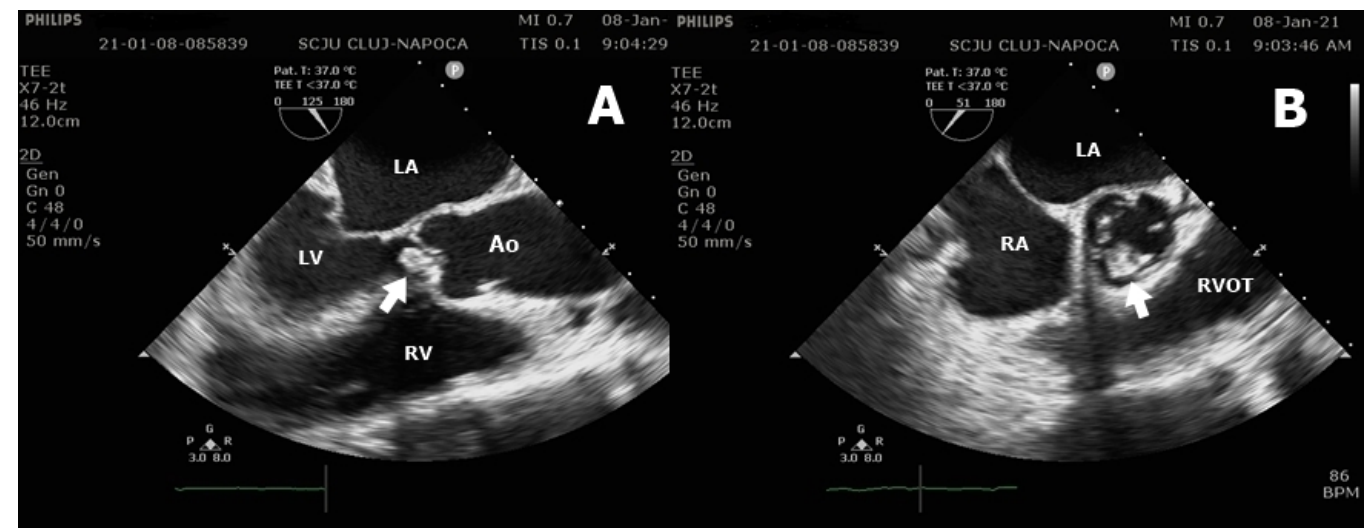

Figure 1. Transesophageal echocardiography: (A) mid esophageal aortic valve long-axis view showing the prolapse of large, mobile vegetation (white arrow) and (B) mid esophageal aortic valve short-axis view with the vegetation (white arrow). $\mathrm{LA}=$ left atrium, $\mathrm{LV}=$ left ventricle, $\mathrm{Ao}=$ aorta, $\mathrm{RV}=$ right ventricle, $\mathrm{RVOT}=$ right ventricular outflow tract

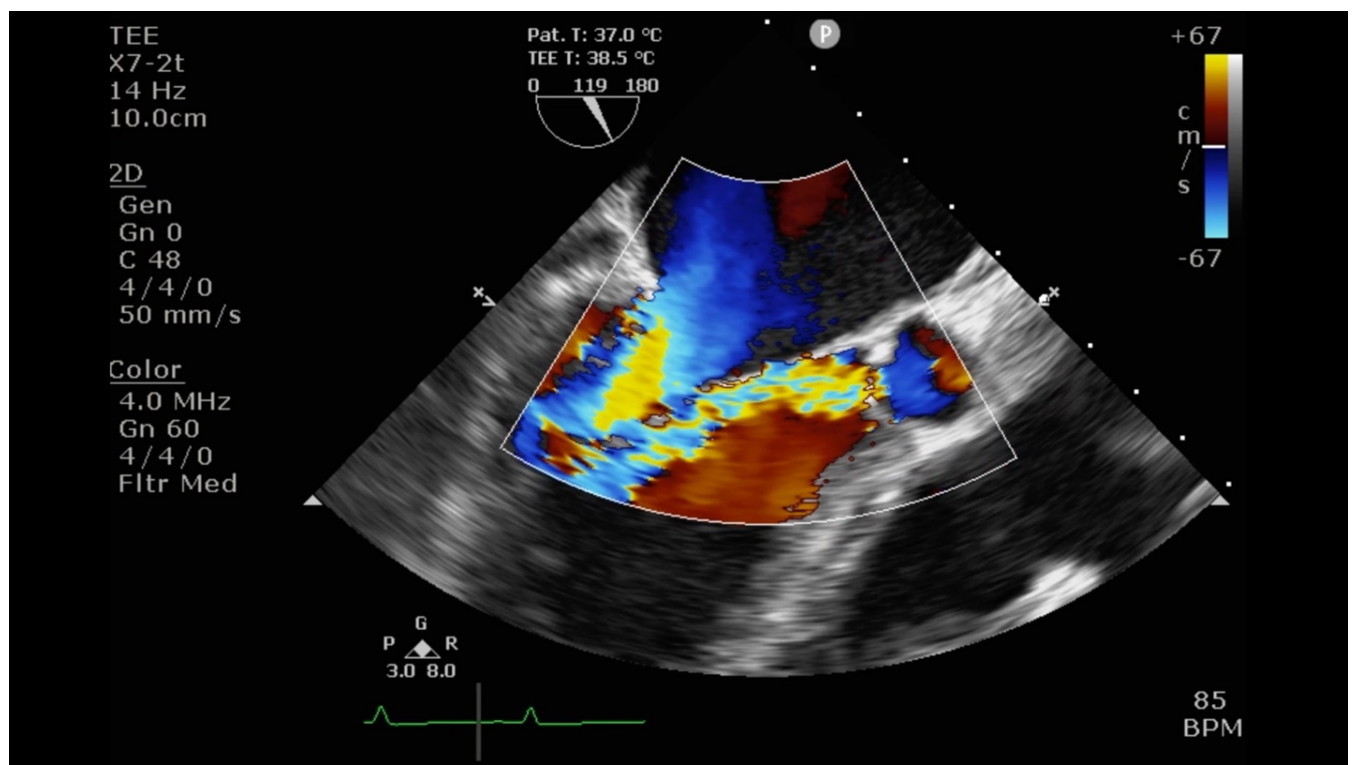

Figure 2: Mid-esophageal aortic valve long-axis view on transesophageal echocardiogram showing severe aortic insufficiency jet with a vena contracta of $6 \mathrm{~mm}$.

An abdominal ultrasound raised the suspicion of splenic abscess and this was confirmed at the non-contrast computed tomography exam that followed (Figure 3). It is known that metastatic infection is most commonly associated in leftsided IE. The computed tomogram of the head was unremarkable.

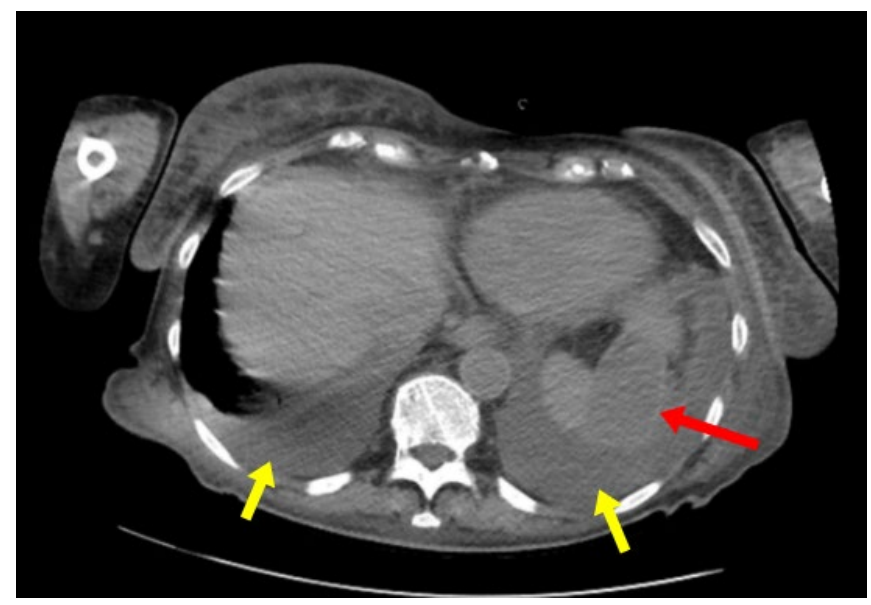

Figure 3: Non-contrast computed tomography scan with $57 \mathrm{~mm}$ splenic abscess (red arrow) and bilateral pleural effusion (yellow arrows). 
The preoperative risk stratification using the STS risk score has shown a $15.51 \%$ risk of mortality, while the EuroScore II predicted a $16 \%$ mortality risk.

The splenectomy was carried out through a left subcostal incision. An enlarged spleen with abscess of $6 \mathrm{~cm}$ on the superior pole showing adhesions to the diaphragm was found intraoperatively. After the procedure the operative field for cardiac surgical intervention was prepared. Median sternotomy was done. Both pleural cavities were opened; $500 \mathrm{ml}$ of serous liquid from the left pleural space and $1300 \mathrm{ml}$ from the right pleural space was evacuated, respectively.

The aortic valve at inspection was tricuspid, the right coronary leaflet of the valve showed total destruction and detachment from the aortic annulus, with multiple voluminous vegetations. An annular abscess at the level of the right coronary/non-coronary cusp commissure was identified, evacuated and closed with Teflon pledget. Through excision and debridement of the infected valvular tissue was performed. A biological St. Jude Trifecta prosthetic valve of number 21 was sutured in the aortic position reinforced with Teflon pledgets. After weaning from cardio-pulmonary bypass (CPB), in order to obtain hemodynamic stability, Noradrenalin 0.089 $\mu \mathrm{g} / \mathrm{kg} / \mathrm{min}$ and Dobutamine with $2.77 \mu \mathrm{g} / \mathrm{kg} / \mathrm{min}$ were initiated. After the first two postoperative hours, due to increased thoracic drainage $(700 \mathrm{ml}$ blood),reintervention for hemostatic control was needed.

On postoperative day 2 the patient became anuric with significantly elevated creatinine and blood urea nitrogen levels, control echocardiography showed severe global hypokinesia, moderate mitral insufficiency and left ventricular ejection fraction (LVEF) of $15 \%$ with normofunctional aortic prosthesis. Continuous renal replacement therapy (CRRT) was initiated for 4 days, with good biological and hemodynamic response. On echocardiography mitral insufficiency was reduced and myocardial contractility improved compared to the previous examination, $\mathrm{LVEF}=45 \%$.

During the postoperative period the patient has presented episodes of atrial fibrillation and was successfully converted to sinus rhythm under Amiodarone infusion. The patient required mechanical ventilation (MV) for 4 days. After weaning from MV with altered neurologic status: psycho-motor agitation, temporal-spatial disorientation, with unmodified findings on repeated cranial computed tomography (CT) scan. The pathologic findings have confirmed the diagnosis of infective endocarditis and the involved pathogen. In the $16^{\text {th }}$ postoperative day the patient was transferred for recovery to a specialized clinic.

The role of cardiac recovery in the elderly after cardiac surgery

The phase I of our patient's recovery took place in a specialized recovery clinic. From a theoretical point of view, the objectives of this phase are: avoiding physical deconditioning, mastering the techniques of performing physical exercises and implementing lifestyle change measures. Respiratory gymnastics, walking and active physical therapy are required. In the Table 2 below, we summarized the recovery program followed by the patient.

Table 2. The patient's recovery program plan.

\begin{tabular}{|c|c|c|c|c|}
\hline $\begin{array}{l}\text { Nr. } \\
\text { Crt }\end{array}$ & $\begin{array}{l}\text { Procedure } \\
\text { code }\end{array}$ & $\begin{array}{l}\text { Procedure } \\
\text { name }\end{array}$ & $\begin{array}{l}\text { Characteristi } \\
\text { cs }\end{array}$ & $\begin{array}{l}\text { Numbe } \\
r \quad \text { of } \\
\text { session } \\
s\end{array}$ \\
\hline 1 & $\begin{array}{l}155 \_668945 \\
\text { S } 1000\end{array}$ & $\begin{array}{l}\text { PARTIAL } \\
\text { MASSAGE } \\
\text { (WARD) }\end{array}$ & $\begin{array}{l}\text { Paravertebral } \\
\text { dorsal-lumbar } \\
\text { spine massage } \\
\text { from sitting } \\
\text { position }\end{array}$ & 10 \\
\hline 2 & $\begin{array}{l}155 \_668945 \\
\text { S } 1002\end{array}$ & $\begin{array}{l}\text { LASER } \\
\text { (WARD) }\end{array}$ & $\begin{array}{l}\text { LASER } \\
\text { shower probe: } \\
\text { ankles and } \\
\text { plants } \\
\text { bilaterally } \\
\text { (neuralgia } \\
\text { program) }\end{array}$ & 10 \\
\hline 3 & $\begin{array}{l}155 \_668945 \\
\text { S } 1001\end{array}$ & $\begin{array}{l}\text { INDIVIDUA } \\
\text { L } \\
\text { PHYSICAL } \\
\text { THERAPY } \\
\text { (WARD) }\end{array}$ & $\begin{array}{l}\text {-twice daily } \\
\text {-recovery for } \\
\text { orthostatism, } \\
\text { walking, } \\
\text { balance } \\
\text {-gentle muscle } \\
\text { tonus } \\
\text { enhancement } \\
\text { in the lower } \\
\text { limbs } \\
\text { NO } \\
\text { OVERUSE } \\
\text { AT } \\
\text { LIMIT } \\
\text { THE OF } \\
\text { EFFORT } \\
\text { TOLERANC } \\
\text { E } \\
\text { THE } \\
\text { ABDOMINA } \\
\text { L MUSCLES } \\
\text { WILL NOT } \\
\text { BE STRESSED }\end{array}$ & 20 \\
\hline
\end{tabular}

At discharge, the patient maintains orthostatism and balance, moves with the walking frame non-stop for a distance of over $100 \mathrm{~m}$.

The phase II recovery will take place in the same clinic, 4 to 6 weeks after the sternal wound healing. 


\section{Discussion}

Infective endocarditis (IE) has a polymorphic clinical presentation because of the acute, subacute or chronic symptoms onset determined by the infective agent. Streptococcus agalactiae, one of the less common causes of IE and it was known to be associated with invasive infections in pregnant women and newborns $(\mathbf{1}, \mathbf{2})$. An increasing number of cases with IE with GBS in elderly patients has been reported in the recent years (3) with high rate of complications and mortality. The underlying comorbidities are important risk factors for S. agalactiae IE. Considering our case, a patient in her eighties with significant abdominal surgical history and multiple medical conditions could be a typical host. Due to the local and systemic complications which could be lifethreatening, a combination of antimicrobial and surgical treatment was considered for a better outcome (6). Aortic valve replacement and splenectomy were performed at the same time, followed by a good recovery. It is known that despite surgical treatment, the overall mortality in GBS IE may reach $40 \%$ in both acute and subacute clinical presentations, similar in percentage to staphylococcal IE (4).

The onset of symptoms and the hyperechoic vegetation seen at TEE suggest a prolonged evolution of the infectious disease. Therefore, local and systemic complications were inevitable. Heart failure is the most common local complication of IE, more likely to appear when the aortic valve is involved. Dyspnea, lower extremity edema and bilateral pleural effusion, signs and symptoms of heart failure, were present since the patient referred to the hospital. Progression of the disease with valvular destruction and acute severe aortic regurgitation caused hemodynamic instability with acute respiratory and heart failure. TTE and TEE were performed to confirm the aortic valve destruction and quantify the severity of aortic regurgitation. A PHT of $157 \mathrm{~ms}$ and vena contracta of $6 \mathrm{~mm}$ were observed. Considering all these, a decision was made to proceed urgently with aortic valve replacement.

In order to comprehensively characterize the morphology and functional status of the cardiac and vascular structures affected by the infectious process, transesophageal echocardiography (TEE) is a noninvasive and cost-effective examination (7). This information is essential in the setting of the surgical approach, considering that a favorable long term outcome depends on the complete removal of the infected tissue followed by the reconstruction or replacement of the excised lesions (7).

A common clinical presentation of patients with IE is acute kidney injury (8). It is worth mentioning the kidney disease progression seen in this case. On admission, as we already mentioned, urinalysis showed microscopic proteinuria and hematuria. The patient's serum creatinine was $2.27 \mathrm{mg} / \mathrm{dL}$ with a GFR of $19 \mathrm{ml} / \mathrm{min} / 1.73 \mathrm{~m} 2$ which considerably increased to $3.8 \mathrm{mg} / \mathrm{dL}$ with GFR of 11 $\mathrm{ml} / \mathrm{min} / 1.73 \mathrm{~m} 2$, despite adequate hydration and diuretic treatment. A combined etiology of acute kidney injury could be discussed. It is known that IE is associated with various forms of kidney disease, ranging from immunemediated glomerulonephritis, renal infarction or abscess to drug-induced acute tubular necrosis or consequent to heart failure with reduced cardiac output. Laboratory tests done after surgery came up negative for $\mathrm{C} 3$ and $\mathrm{C} 4$ complement and circulating immune complexes and there was no evidence of renal infarction or abscess. Serum creatinine dropped to $1.23 \mathrm{mg} / \mathrm{dL}$ in the third postoperative day, hinting to a prerenal cause of acute kidney injury (Figure 4).

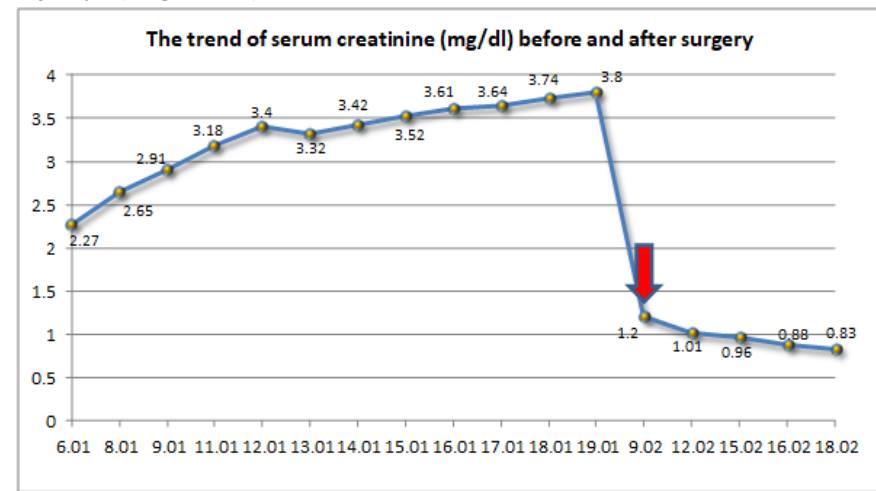

Figure 4. The trend of serum creatinine. There was a significant improvement of the renal function noted after surgery (red arrow) when compared with the values from before surgery.

Taking into consideration the patient's age and frailty, we considered the risk of performing two surgical interventions separated by a short period of time to be much higher than the benefits; accordingly, we decided to perform the splenectomy at the same time with the replacement of the affected valve.

The postoperative result was favorable, with a more difficult evolution but with the complete recovery of the patient. This simultaneous approach to splenectomy and valve replacement has not been used until today in our country, so we consider our intervention as a national premiere.

Systemic emboli might occur in up to $50 \%$ of patients with IE. Surgery already has an established role in the management of IE across a wide range of patients, a role that increases as the complexity of patients with this difficult condition rises (9).

In such cases with high risk, complex patients, a strong collaboration between all specialties is needed to overcome all of the limitations of the patient's comorbidities (10).

Recovery after cardiac surgery is one of the most important indications of physical training. This includes patients post-coronary artery bypass grafting, after valve 
prosthetic replacement, after surgery for congenital diseases and after heart transplantation (11). Patients receiving cardiac surgery usually have severe heart disease, which leads to a significant decrease in cardiac output and low exercise capacity. Many cardiac surgery patients end up with exertion if not equal to the sedentary individuals in their age group, often superior to them (11).

Phase I starts from the first days postoperatively in the intensive care units and continues post-intensive care. Days 2-5 postoperatively include mobilization, progressive movements, and daily exercise (12).

Early mobilization is particularly important in avoiding immobility and cardiac deconditioning (12). Of course, early mobilization is not possible in the presence of associated severe heart failure.

Native valve infectious endocarditis is a relatively rare complication, with an incidence of 2-10 cases per 100,000 patients / year. In patients with infectious endocarditis, involvement of the spleen in infection is rare, but is a well-known complication. Splenic abscess is associated with an increased degree of morbidity and mortality due to complications such as abscess rupture and / or spread of bacteremia. The need to remove the source of extracardiac infection source before repairing or replacing the infected valve is accepted by most surgeons. However, the timing and indication for the operation remains a controversial topic.

Currently, there are two generally accepted therapeutic approaches, namely, the first suggesting the replacement of the involved valve, followed by antibiotic therapy and CT follow-up of the abdomen, and another recommending valve surgery and splenectomy either simultaneously or in stages. However, it should be borne in mind that the splenic abscess can contribute to the spread of the infection, its rupture can occur at any time, and this complication can be fatal for the patient. There are studies that show a $100 \%$ mortality in those without splenectomy, and a survival of $82 \%$ in those with splenectomy, which clearly shows that the removal of the spleen has a positive impact in reducing the risk of superinfection of the implanted valve.

\section{Conclusions}

Despite the fact that mortality in Group B Streptococcus endocarditis is $40 \%$, a combined medical and surgical strategy individualized to the specific situation of each patient have led to a positive outcome in a number of cases. We present such a combined treatment approach in a case of complicated infective endocarditis with metastatic spleen abscess in an elderly patient. The aortic valve replacement and splenectomy were performed at the same time.

\section{Informed consent}

An informed consent was obtained from the patient participating in the study.

\section{Conflicts of interest}

All authors have nothing to disclose.

\section{Ethical Policy}

During this study, the procedures followed were in accordance with the ethical standards of the responsible committee on human experimentation (institutional and national) and with the Helsinki Committee for Human Rights. Consequently, the study obtained the $\backslash$ approval from The Ethical Commission of our institution.

\section{References}

1. Ya'qoub L, Rehan L, Parikh S, Enriquez J. Streptococcus Agalactiae Infective Endocarditis in a Healthy Middle-aged Man: Uncommon but Life-threatening. Cureus. 2018;10(5):e2632.

2. Schrag SJ, Zywicki S, Farley MM, Reingold AL, Harrison LH, Lefkowitz LB, et al. Group B streptococcal disease in the era of intrapartum antibiotic prophylaxis. $\mathrm{N}$ Engl $\mathrm{J}$ Med. 2000;342(1):15-20.

3. Sambola A, Miro JM, Tornos MP, Almirante B, MorenoTorrico A, Gurgui M, et al. Streptococcus agalactiae infective endocarditis: analysis of 30 cases and review of the literature, 1962-1998. Clin Infect Dis. 2002;34(12):1576-84.

4. Scully BE, Spriggs D, Neu HC. Streptococcus agalactiae (group B) endocarditis--a description of twelve cases and review of the literature. Infection. 1987;15(3):169-76.

5. Farley MM, Harvey RC, Stull T, Smith JD, Schuchat A, Wenger JD, et al. A population-based assessment of invasive disease due to group B Streptococcus in nonpregnant adults. N Engl J Med. 1993;328(25):1807-11.

6. Tchana-Sato V, Hans G, Frippiat F, Zekhnini I, Dulgheru R, Bruls $\mathrm{S}$, et al. Streptococcus agalactiae infective endocarditis: analysis of eight surgical cases from a single centre. Infect Dis (Lond). 2021;53(3):189-95.

7. Molnar A, Sacui D, Manole S, Radulescu A, Beyer R. The value of transthoracic and transesophageal echocardiography for the diagnosis of the native aortic infective endocarditis valve complications: a case report and literature review. Med $\begin{array}{lllll}\text { Ultrason. } 2016 ; & 18 & \text { (2): } 253-6 . & \text { DOI: }\end{array}$ 10.11152/mu.2013.2066.182.ttr.

8. Boils CL, Nasr SH, Walker PD, Couser WG, Larsen CP. Update on endocarditis-associated glomerulonephritis. Kidney Int. 2015;87(6):1241-9.

9. Molnar A, Beyer R, Florian S, Muresanu DF, Trifan C, Muresan I, et al. Drainage of cerebral abscesses prior to valve replacement in stable patients with acute left-sided infective endocarditis. CNS \& Neurological Disorders Drug Targets. 2015;14(4):534-9.

DOI:

10.2174/1871527314666150317224921.

10. Moldovan H, Popescu D, Buliga T, Filip A, Antoniac I, Gheorghita D, Molnar A. Gastric adenocarcinoma associated with acute endocarditis of the aortic valve and coronary artery disease in a 61-year-old male with multiple comorbiditiescombined surgical management-Case report. Medicina. 2019; 55: 242. DOI:10.3390/medicina55060242.

11. Zdrenghea D. Recuperare si preventie cardiovasculara. Clusium; 2008.

12. Zdrenghea D, Pop D, Mitu F. Cardiologie preventiva si recuperare cardiovasculara. Clusium; 2020. 